NASA Technical Memorandum 107196

\title{
A Numerical Investigation of the Startup Transient in a Wave Rotor
}

Daniel E. Paxson

Lewis Research Center

Cleveland, Ohio

Prepared for the

41st Turbo Expo '96

sponsored by the International Gas Turbine Institute of

the American Society of Mechanical Engineers

Birmingham, United Kingdom, June 10-13, 1996

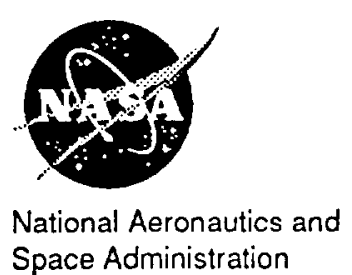

Space Administration 
$\therefore$ 


\title{
A NUMERICAL INVESTIGATION OF THE STARTUP TRANSIENT IN A WAVE ROTOR
}

\author{
Daniel E. Paxson \\ NASA Lewis Research Center \\ Cleveland, Ohio, USA
}

\begin{abstract}
The startup process is investigated for a hypothetical four-port wave rotor, envisioned as a topping cycle for a small gas turbine engine. The investigation is conducted numerically using a multi-passage, onedimensional CFD based wave rotor simulation in combination with lumped volume models for the combustor, exhaust valve plenum, and rotor center cavity components. The simulation is described and several startup transients are presented which illustrate potential difficulties for the specific cycle design investigated. In particular it is observed that, prior to combustor light-off, or just after, the flow through the combustor loop is reversed from the design direction. The phenomenon is demonstrated and several possible modifications techniques are discussed which avoid or overcome the problem.
\end{abstract}

\section{INTRODUCTION}

The wave rotor is currently being investigated for use as a topping cycle for future gas turbine engines. The device, shown schematically in Fig. 1, uses gasdynamic waves to transfer energy directly to and from the working fluid through which the waves travel. Typically, it consists of a series of constant area passages that rotate about an axis. Through rotation, the ends of the passages are periodically exposed to various circumferentially arranged ports which initiate the travelling waves within the passages. The particular location of the ports determines the thermodynamic cycle of the working fluid. When used as a gas turbine topping cycle, the gas path is such that each passage of the wave rotor is periodically exposed to both hot and cold flow. The mean temperature of the rotor material is therefore considerably below the peak cycle temperature. This feature, combined with the relatively efficient nature of unsteady gasdynamic work transfer, and low rotational speed make the wave rotor a strong potential candidate for achieving high peak cycle temperatures and pressures without resorting to advanced materials. Steady state simulations of the wave rotor, in a topping cycle environment, using validated numerical codes, have shown favorable

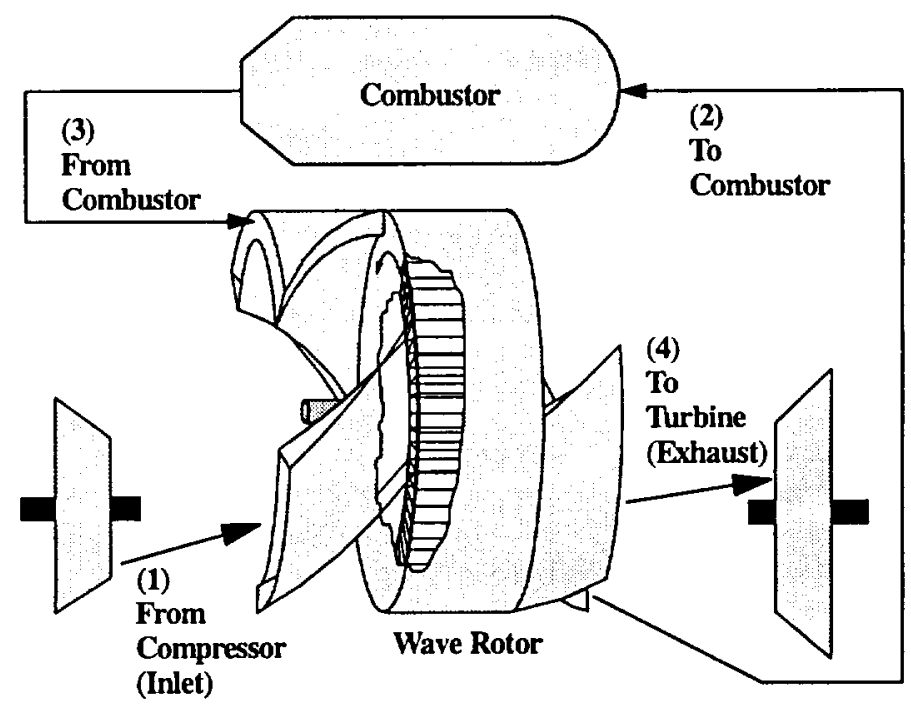

Figure 1 Wave rotor schematic

performance both on and off-design (Welch et. al., 1995). Promising experimental results have also been demonstrated by Klapproth (1960), Moritz (1985), and Thayer (1985). Unsteady simulations (i.e. time dependant boundary conditions) have indicated that the wave rotor responds very rapidly to transient input, and is quite robust even when the magnitudes of the transient input perturbations are severe (Paxson, 1995a).

In most of the published analytical, numerical, and experimental literature however, it is assumed that the wave rotor is already running. That is to say, the simulations (or experiments) are approximately centered about the design point. The question of how to actually start a wave rotor is an important one and is particularly relevant to the NASA Lewis Research Center, where a prototype four-port wave rotor is 
presently being fabricated for testing. It is not obvious what the wave patterns look like in the extreme off-design regime of startup, or even if a wave pattern can easily be established. In the literature on past wave rotor experiments, little has been written regarding the starting procedure; however, several publications suggest that it was not trivial. Klapproth (1960) mentioned the use of a propane pilot burner in order to accurately control the temperature in the combustor loop during startup. Zhender et. al. (1989) described a bypass valve for starting a diesel engine equipped with a wave rotor super-charging system. Moritz (1965-1970) suggested that a wave rotor topped gas turbine may not start conveniently, although no explanation was given for this conclusion.

The present paper examines the starting process for a particular wave rotor using a numerical wave rotor simulation (Paxson, 1995a) as the test environment. It is noted that there are many conceivable wave rotor cycles for many types of applications and that the starting process may vary from one cycle to the next. The focus in this paper is on a hypothetical through-flow, four-por wave rotor which would be used as a topping cycle for a small gas turbine engine. The term 'through-flow' refers to the fact that the flow from the upstream compressor travels from one end of the wave rotor to the other before being sent to the combustor (Welch et. al., 1995).

In the pages which follow, details of the wave rotor under investigation will first be presented. A brief description of the numerical simulation will then be given along with the associated simplifying assumptions. Following this, several hypothetical wave rotor start transients will be shown and discussed. The numerical results will show that starting scenarios exist where the flow in the combustor loop is reversed from the design direction. It will also be shown however, that with careful control of fuel flow and rotor speed, or with some augmentation to the rotor design such as the addition of an injection valve in the combustor loop or modification of the timing in the port leading from the combustor, this phenomenon can be avoided or overcome.

\section{WAVE ROTOR DESCRIPTION}

The specific dimensions and design point operating performance of the wave rotor under consideration may be found in Table 1 . The gas turbine engine which it is intended to top has a compressor pressure ratio of approximately 8 at the design point. The wave rotor ducts are placed at angles to the axis of rotation which allow it to be self-driven under normal operating conditions; however, for the startup operations to be examined it is assumed that an independent drive motor is available. The rotor passages are aligned with the axis of rotation, and have constant trapezoidal cross section. The placement of the ports, their angles, and a computed design point wave pattern for this wave rotor may be seen in Fig. 2. This figure shows the time averaged (averaged over the time required for one rotor passage to traverse a given point) contours of gas density in the passages for one cycle of the wave rotor. The contours were computed using a one dimensional CFD simulation code which follows a single passage of the wave rotor as it rotates past the various ducts (Paxson, 1995b, c). The numerical spacing in the passage was $2 \%$ of the length. The time step was set at $0.6 \%$ of the time required for a sound wave at the design point inlet stagnation temperature to travel from one end of a passage to the other. The through-flow nature of the cycle can clearly be seen in this figure by noting the movement of the inlet gas from left to right. It is interesting to note as an aside that this figure illustrates a recirculation of hot gas through the combustor at the design point. That is, for this four-port through-flow cycle, there is more gas travelling to and from the combustor than there is from the inlet.
Table 1 Wave rotor dimensions and design point performance

\begin{tabular}{lll}
\hline \hline Mean Rotor Radius & $8.15 \mathrm{~cm}$. & $(3.2 \mathrm{in})$. \\
Rotor Length & $15.24 \mathrm{~cm}$ & $(6.0 \mathrm{in})$. \\
Rotor Passage Height & $2.18 \mathrm{~cm}$. & $(0.86 \mathrm{in})$. \\
Rotational Speed & $16800 \mathrm{rpm}$ & \\
Cycles $/$ Revolution & 2 & \\
Number of Passages & 52 & \\
Mass Flow Rate & $2.3 \mathrm{~kg} / \mathrm{s}$ & $(5.0 \mathrm{lbm} / \mathrm{s})$ \\
$\mathrm{P}_{4} / \mathrm{P}_{1}$ (Fig. 1 or 2$)$ & 1.23 & \\
$\mathrm{~T}_{4} / \mathrm{T}_{1}$ & 2.21 & \\
\hline \hline
\end{tabular}

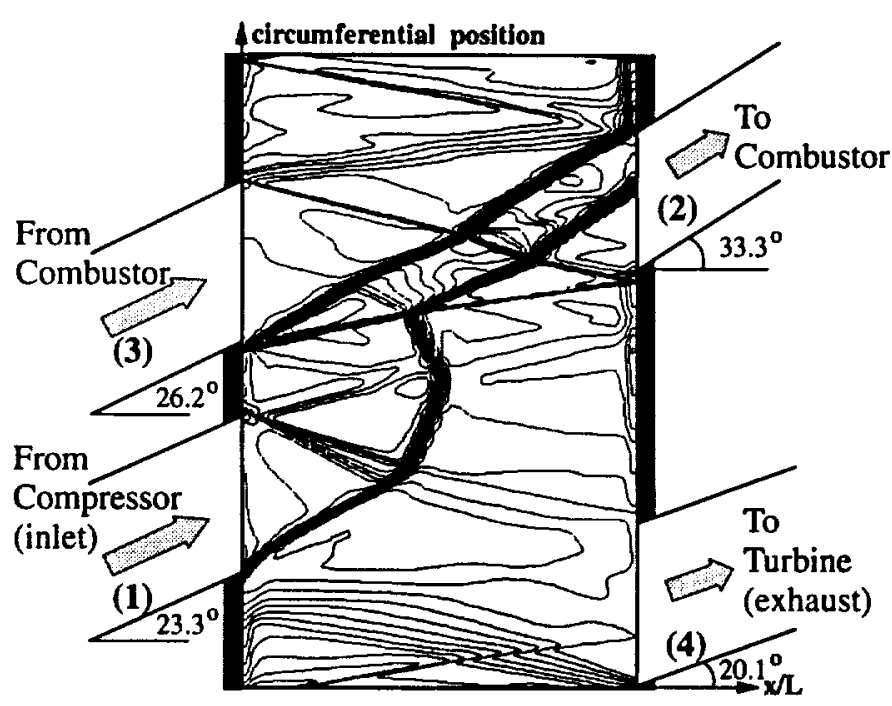

Figure 2 Port placement and time averaged design point wave pattern

\section{NUMERICAL SIMULATION DESCRIPTION}

The wave rotor simulation used in this investigation has been described in detail elsewhere (Paxson, 1995a). As such, only a brief verbal description will be provided here in order to maximize space for presentation of results and discussion. The simulation 'layout' is shown schematically in Fig. 3. The gasdymics in each passage of the wave rotor itself are modeled using a high resolution one-dimensional, perfect gas CFD code which integrates the Euler equations with a source term. The source term accounts for losses due to boundary layer viscous effects, heal transfer to and from the passage walls, and leakage to and from the center cavity inside the rotor housing. Boundary conditions for the port regions of the code are supplied as stagnation states. These are either provided directly by the user, as in the case of the port leading from the upstream compressor, or calculated by several lumped volume models of the extemal components. These components consist of the combustor, which couples two of the ports (ports 2 and 3 ), and the plenum linking the exhaust valve to the exhaust port (port 4). The rotor center cavity, to and from which leakage occurs, is also modelled as a lumped volume component. This cavity is comprised of the empty space inside the rotor housing which is not occupied by the rotor itself. The 


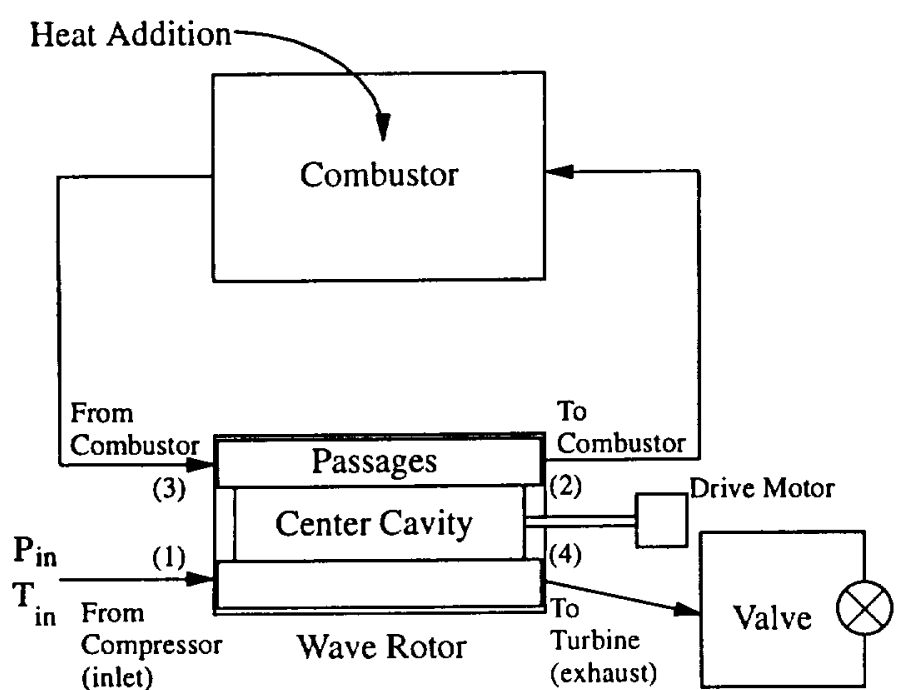

Figure 3 Simulation schematic showing modelled components

pressure difference between the cavity and the gas in the passages, together with the specified gap between rotor and endwalls, governs the leakage flow via a source term in the first and last computational cell of each passage. In this paper, the term 'lumped volume' means that the kinetic energy of the flows in the components is assumed low. Thus, they are modelled using only mass and energy conservation equations. The energy equation for the combustor component contains a term which allows for external heat addition. This term simulates energy into the system which is derived from burning the fuel. It is noted that such a model does not account for the added mass of fuel to the system, nor does it account for the delay between fuel injection and heat release. It does not seem likely however, that either of these shortcomings will significantly affect the results to be presented. The rate of heat addition (i.e. simulated fuel flow), is assumed to be externally controlled. Additionally, the area of the exhaust valve, which simulates the downstream turbine, is assumed externally controlled; however, in this study, the area was held constant at the value prescribed by the design point operating point.

The stagnation boundary conditions supplied by the user or component models are used by each passage in the CFD code to determine the state of the so called 'image' cell at the next instant of time. The code is capable of assessing whether a given condition will lead to inflow or outflow in a given passage. This allows robust operation of the simulation even in far off-design conditions where a portion of the flow in a given port may be into the rotor and a portion out of the rotor. For outflow conditions, only the boundary pressure is used, and although specified as stagnation, it is treated as a static value. For inflow conditions some accounting is made if the flow in the duct is not aligned with the passage (i.e. shaft work into the system). For both inflow and outflow conditions, accounting is made at the boundaries for the effects on the flow of those passages which are only partially opened to a port; so called finite opening effects. The ducts leading to and from the ports are assumed loss free (isentropic); however, a constant area mixing calculation is used in outflow ports to account for losses due to nonuniformities in the flow and a stagnation pressure loss proportional to the square of the mass flow is imposed on the flow going through the combustor loop. No accounting is made for losses incurred due to the finite thickness of the passage walls.

In order to avoid undue demand on the computing resources a rather large numerical spacing was used for the simulation results to be presented. Unless otherwise stated, each numerical cell of each passage in the wave rotor code was $10 \%$ of the length. The corresponding time step was set at $5 \%$ of the time required for a sound wave at the design point inlet stagnation temperature to travel from one end of a passage to the other. Such crude grid spacing obviously leads to inaccuracies in the results; however, given the extreme off-design conditions in which the code is nunning, the errors incurred from crude grid spacing are likely no worse than those which arise from several of the simplifying assumptions made in the model (i.e. one dimensional flow, loss free ducts, uniform static pressure across outflow ports, etc.) and none are sufficiently severe as to invalidate the results.

It is noted that the simulation described above does not include a model for the upstream compressor. The stagnation conditions in the inlet are simply specified. In reality, there would be a high degree of dynamic interaction between the compressor and the wave rotor, including possible system instabilities. Investigations into these sorts of interactions are planned.

\section{SIMULATION RESULTS}

The results to be presented below are divided into several simulation scenarios which illustrate various aspects of the starting process. Each scenario is labelled with a letter and a brief descriptor. In the subsequent discussion, the various scenarios are referred to by their letter. Although many system variables are informative, only the port mass flow rates, plenum pressures, and controlled variables (i.e. rotor speed and heat addition rate) will be presented in the following time trace plots. The mass flow rates are normalized by the design point inlet mass flow rate. The pressures are normalized using atmospheric pressure. The rotor speed is shown as a fraction of the design point value. The data output interval from the simulations was short enough to capture fluctuations in system variables caused by passage opening and closing in the ports; however, the data shown in the time traces has been smoothed in order to eliminate this effect. For all of the simulation scenarios the pressure downstream of the exhaust valve was held at the standard atmospheric value.

\section{Scenario A: Initial Spoolup Without Heat Addition}

The initial phase of the start process establishes flow through the rotor with no heat added to the combustor. In an engine environment this would presumably occur by simultaneously spooling the upstream compressor and wave rotor up to some low speed. Figure 3 shows the simulated results of such a process. The spooling of the upstream compressor was simulated using a linear rise in the wave rotor inlet stagnation pressure from atmospheric to $30 \%$ above atmospheric over the first 1.0 second period of the simulation. After this it was held constant. The corresponding inlet stagnation temperature was calculated assuming a compressor efficiency of 0.7 . The rotor was linearly accelerated from 1.8 to $32 \%$ of the design rotational speed $(2.6-44 \%$ of the corrected rotational speed) over the initial 2.0 seconds of simulation time after which it was held constant. Figure 4 shows time traces of the four port mass flow rates, and the combustor and exhaust plenum pressures. Also shown for reference are the inlet pressure and the rotor speed schedules. The rotor acceleration was chosen to be slow enough such that all of the flow quantities were essentially in equilibrium. Thus, although the rotor 

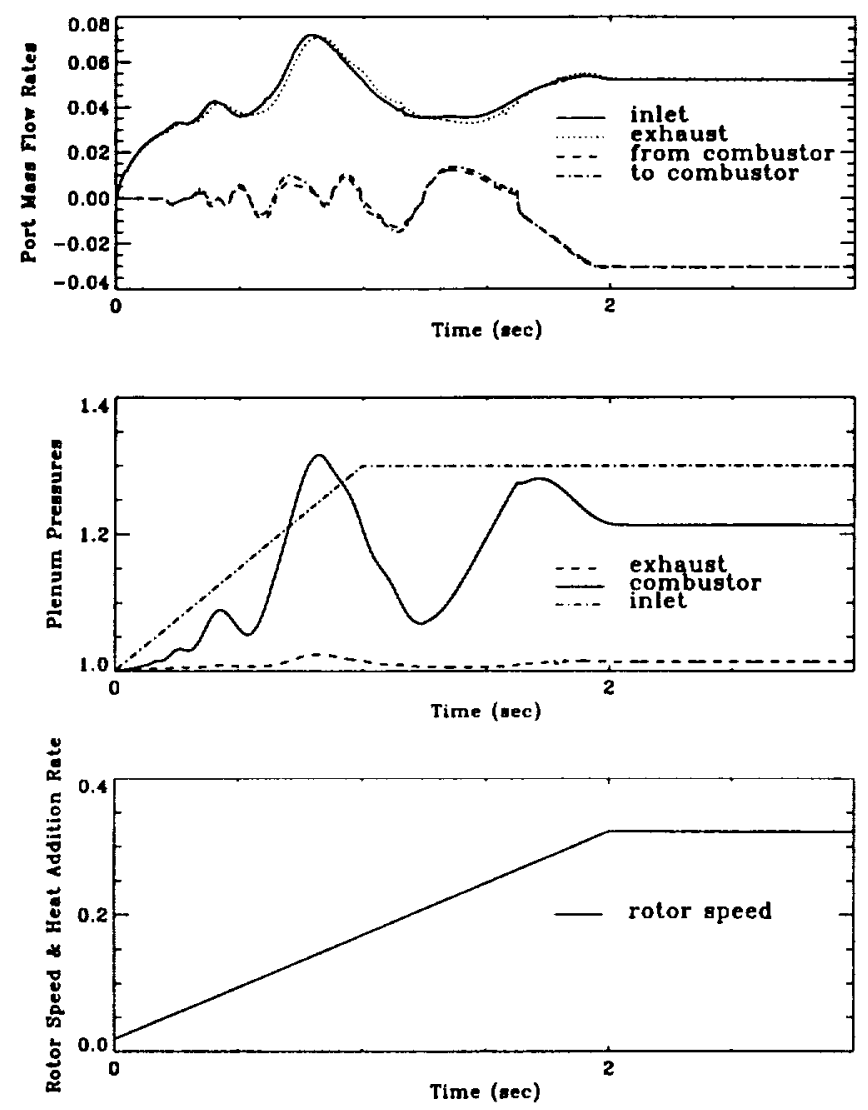

Figure 4 Time traces of flow quantities for Scenario A: Initial Spoolup Without Heat Addition

speed was increasing, the flow quantities at any instant of time are essentially steady state results. This may be seen by noting that the inlet and exhaust port mass flow rates are nearly coincident as are those leading to and from the combustor. Furthermore, it can be seen that after the initial 2.0 second rotor acceleration period the flow quantities do not change. The salient feature of this plot is the apparently cyclic oscillation between forward and reversed flow in the combustor loop. The waves that exist in the rotor are quite weak during the startup transient; however, examination of the computed wave diagrams indicates that these oscillations are related to the number of reflections, and their location relative to the ports leading to and from the combustor, of the weak shock which is generated by the closing of the exhaust port (port 4 in Fig.3).

\section{Scenario Bi Contlnued Constant Rotor Acceleration Followed by Ramp Heat Addition Rate Increase}

The rotor speed from Scenario $A$ at which the largest forward combustor loop flow occurred was used as the starting point for all of the remaining simulations to be discussed. This speed was found to be approximately $23 \%$ of the design speed. The upstream compressor pressure ratio was maintained at 1.3. Figure 5 shows the results of a further constant rotor acceleration followed by combustor heat addition.
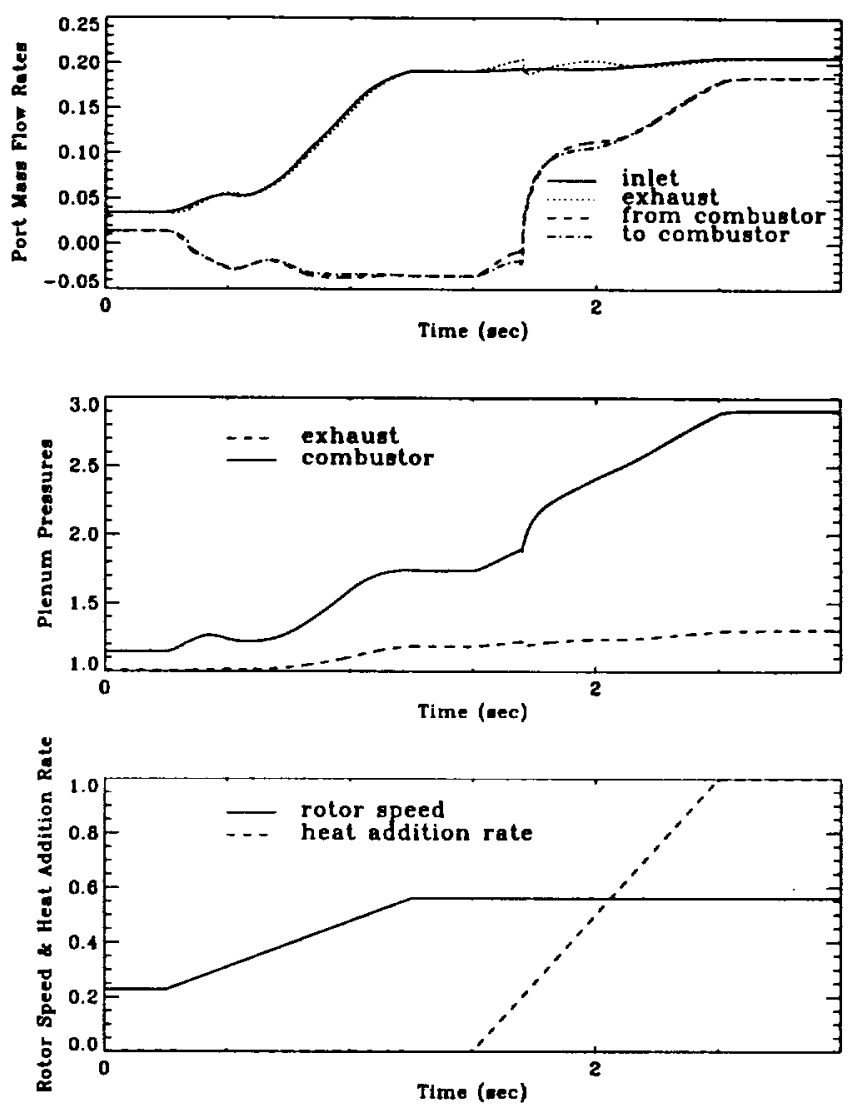

Figure 5 Time traces of flow quantities for Scenario B: Continued Rotor Acceleration Followed by Ramp Heat Addition Rate Increase

The rotor speed was held constant for the first $0.25 \mathrm{sec}$. then increased over a 1.0 second period to $56 \%$ of design speed where it was maintained for the remainder of the simulation. At $1.5 \mathrm{sec}$. the heat addition rate (i.e. fuel flow rate) was increased linearly from 0.0 to $4.9 \%$ of the design point value over a 1.0 second interval after which it was held constant. The final values of rotor speed and heat addition rate were chosen because, in steady state, they result in a favorable idle position where there is zero net torque on the rotor (i.e. no drive motor necessary) and a pressure ratio across the wave rotor, $P_{4} / P_{1}$ slightly above unity. The plotted quantities of Fig. 5 are the same as those in Fig. 4 except the constant inlet pressure is not shown and the heat addition rate is presented as a fraction of the final idle value. It can be seen in this plot that, prior to the addition of any heat, further increases in rotor speed do not yield forward combustor loop flows. In fact, the flow through the combustor loop becomes more negative as rotor speed is increased above the $23 \%$ of design speed setting. It is interesting to note that prior to any heat addition, the inlet mass flow rate increases steeply with rotor speed. This is due to the establishing a favorable wave pattern (i.e. waves terminating and originating at port opening and closing positions) in the inlet and exhaust port section of the wave rotor. It is also interesting that, once the idle rotor speed has been reached, the inlet and exhaust mass flow rates are relatively insensitive to the subsequent and large changes in the combustor loop mass flow rates. As the heat addition rate is 
increased (from zero) it is seen that the combustor pressure rises and the flow through the combustor loop approaches zero. At approximately 1.7 seconds there is an abrupt change in flow direction, which is followed in turn with a rapid increase in the mass flow rate. Once forward flow has been established in the combustor loop, the system approaches the steady state idle in a smooth fashion.

For reference, the steady state idle wave pattern is shown in Fig. 6 in the form of time averaged density contours as was done for Fig. 1, Figure 6 was made using the same single passage (steady state) code described for Fig. 1. The numerical grid spacing was again, $2 \%$ of the passage length. The time step was set at $0.1 \%$ of the time required for a sound wave at the design point inlet stagnation temperature to travel from one end of a passage to the other.

The starting sequence described above (rotor acceleration followed by heat addition) is undesirable due to the initially reversed combustor loop flow. It is not practical to light a conventional combustor with reversed flow. Thus, this scenario would require some sort of auxiliary pilot flame, or other heat source, in the combustor which would be insensitive to flow direction.

\section{Scenario C: Simultaneous Constant Rotor Acceleration and Ramp Heat Addition Rate Increase}

Although the results are not presented here due to space constraints, it was found that reversed flow in the combustor loop also occurred when heat was added to the system prior to rotor acceleration (e.g. essentially the opposite sequence of Scenario B). When both the rotor speed and the heat addition rate were simultaneously increased however, it was found that the startup sequence proceeded without reversed combustor loop flow. The results are shown in Fig. 7. The rates and limits are the same as in Scenario B; however, both were initiated at $0.25 \mathrm{sec}$. As with the previous two scenarios, the system appears to be operating in a nearly steady state fashion throughout most of the simulation. This suggests that the 4 port, through-flow wave rotor may be started in general by coupling the heat addition rate (i.e. fuel flow) to the rotor speed, although the optimal relationship will not necessarily be linear as is shown here. It is doubtful however, that conventional combustors have this sort of fine control capability in the very low fuel flow range of operation. Thus, it is probable that the start sequence described here would again require an auxiliary heat source.

\section{Scenario D: Step Minimum Heat Addition Rate and Simultaneous Rotor Acceleration}

A more appropriate representation for the heat addition rate during the early portion of startup would probably be a step increase to some fraction of the idle value. This would simulate the initial combustor light-off. Following this would be a ramp increase (linear or otherwise) up to idle. Although the results are again not shown due to space limitations, it was found that, starting from the same steady state operating point as Scenario B, without increasing the rotor speed, a step increase of the heat addition rate to less than $11 \%$ of the idle value $(0.6 \%$ of design) was enough to cause flow reversal in the combustor loop. Presumably, there is some minimum fuel flow level required in order to establish a flame in the combustor and it is assumed here to be larger than $0.6 \%$ of design. For this study, a minimum value for the heat addition rate of $1.2 \%$ of design ( $25 \%$ of the idle value) was chosen for the initial step to simulate combustor light-off. What was sought was the minimum

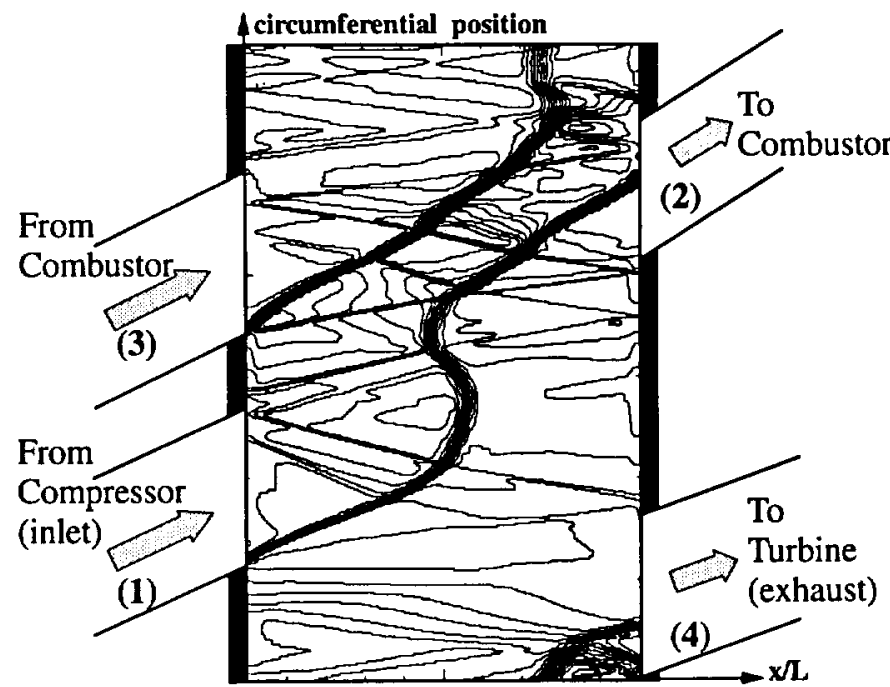

Figure 6 Idle point time averaged wave pattern shown as contours of density
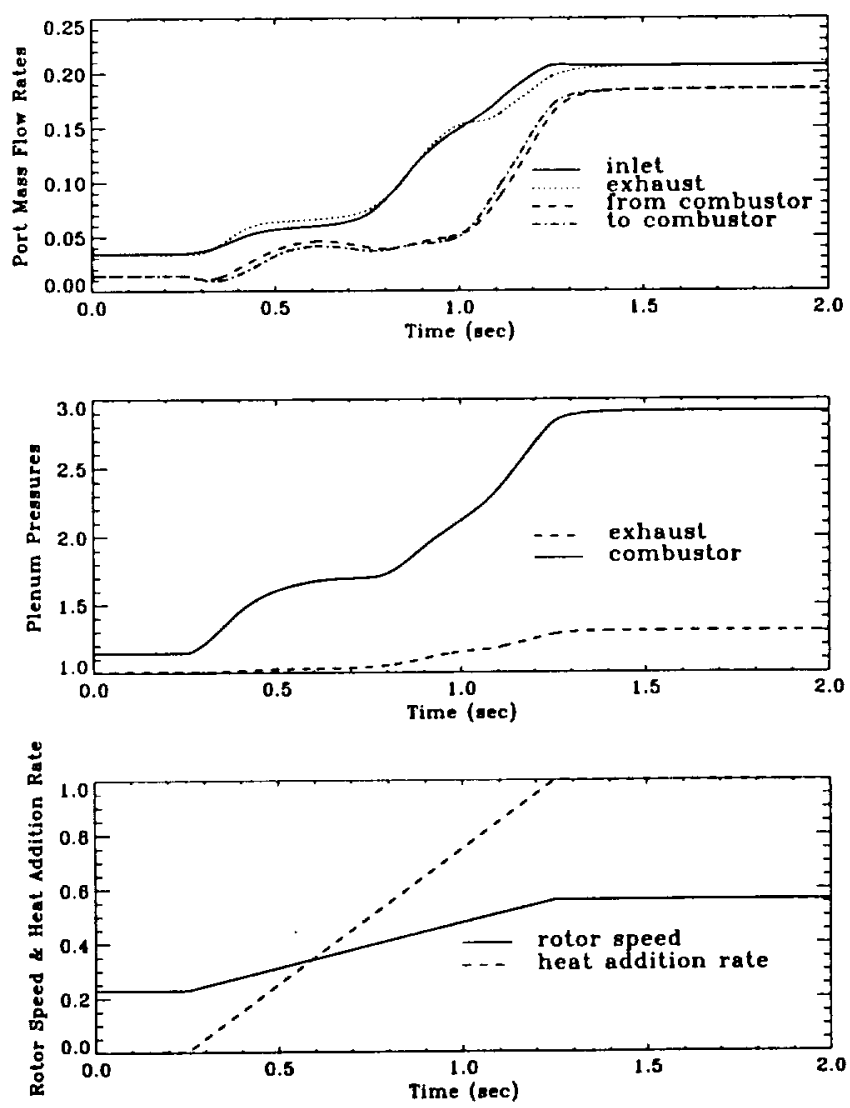

Figure 7 Time traces of flow quantities for Scenario C: Simultaneous Constant Rotor Acceleration and Ramp Heat Addition Rate Increase 


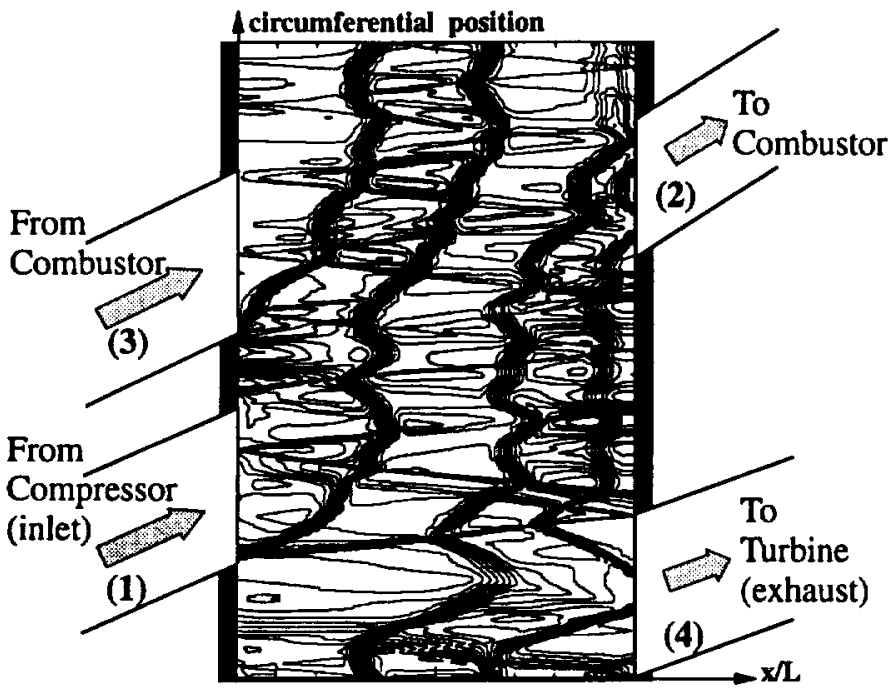

Figure 8 Post lightoff time averaged wave pattern shown as contours of density
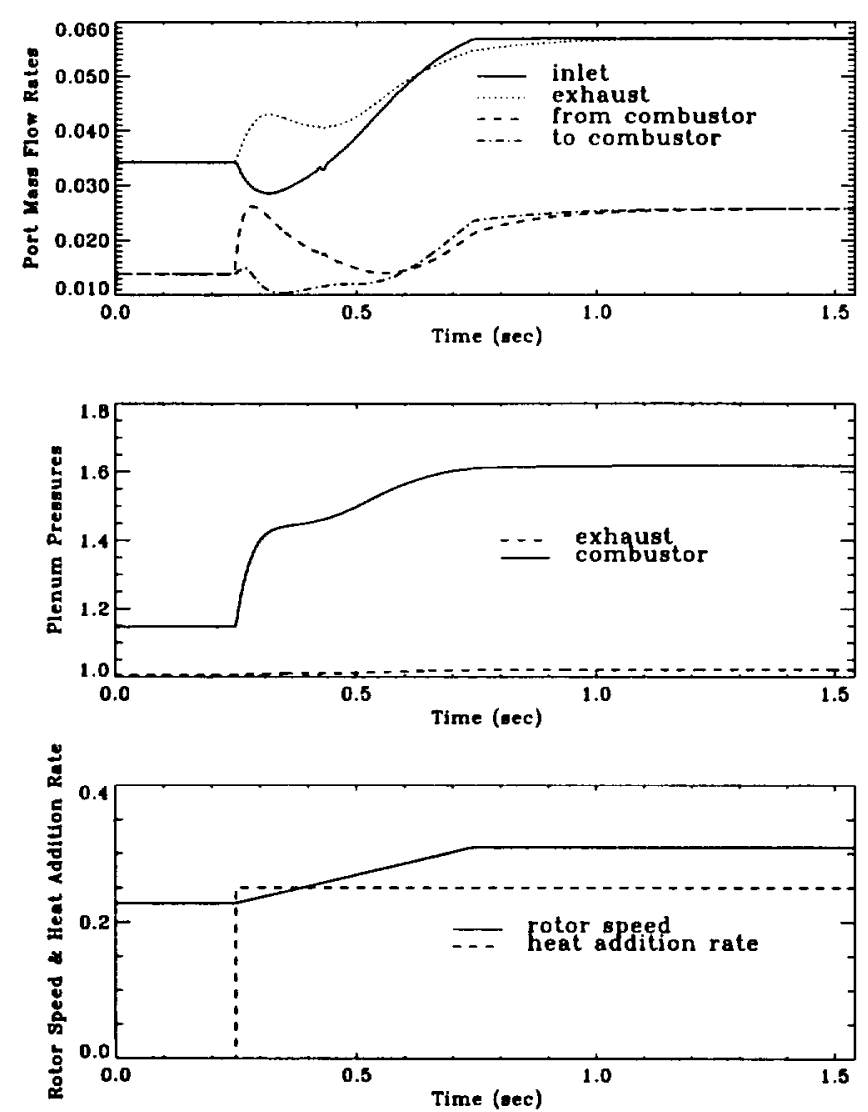

Figure 9 Time traces of flow quantities for Scenario D: Step Minimum Heat Addition Rate and Simultaneous Rotor Acceleration rotor acceleration which would maintain forward flow through the combustor. Due to the simplicity of the combustor model, no consideration was given as to whether the flow of fresh air was sufficient to maintain a flame. Assuming Fig. 7 to be essentially steady state (though not optimal), the final rotor speed was chosen which corresponded to the $1.2 \%$ of design step heat addition rate. This rotor speed was found to be approximately $31 \%$ of design value. The wave diagram for this steady state 'post lightoff operating point is shown in Fig. 8 using the same technique as Fig. 6. A true minimization process would have involved many simulation runs. The objective in this paper is simply to demonstrate a concept. As such the rotor acceleration minimization process was considered complete when a value less than or equal to that used in Fig. 7 was obtained which would maintain forward flow through the combustor loop. It was found that a constant rotor acceleration rate from $23-31 \%$ of design speed over a 0.49 second interval $\left(292.7 \mathrm{rad} / \mathrm{s}^{2}\right)$ would fit this criteria. The results are shown in Fig. 9. The acceleration and step rise in heat addition were commenced simultaneously at $0.25 \mathrm{sec}$. The plotted quantities are the same as in Fig. 7. An estimate of the torque required for the plotted rotor acceleration yielded a value of approximately $18.0 \mathrm{~N}-\mathrm{m}(13.3 \mathrm{ft}-\mathrm{lbf})$. This was obtained using an approximation for the rotor moment of inertia and an assumed material density of $7860 \mathrm{~kg} / \mathrm{m}^{3}$. The bearings were assumed frictionless and drag between rotor and housing was assumed negligible. The instantaneous power requirement of the starting motor during the acceleration was thus found to vary linearly from 7.3 to $9.8 \mathrm{~kW}$ (9.7-13.0 h.p.). These are large values for an engine of this size, particularly in light of what is already required by starter motor for the upstream compressor. As such, Scenario D would not be practical for an aircraft engine but may be acceptable in other applications.

\section{Scenario E: Combustor Plenum Mass Iniection}

The investigation thus far has centered around starting methods which do not require augmentation of the wave rotor other than providing a drive motor. It is worthwhile to consider now some simple augmentations to the wave rotor design which might make the starting process somewhat more robust. One possibility is to add bleed and/or injection valves. Another possibility is to allow for small changes to the timing of one or several ports. These techniques will be explored in the following two scenarios.

For the injection or bleed technique the valves could be placed either in plenum locations or strategically on the rotor endwalls. In the latter location, of course, they are essentially acting as extra ports and thus, the wave rotor is no longer a four-port device. In this paper, only the results of plenum injection will be presented. In particular, a valve was added to the combustor plenum, similar to the exhaust port valve, which had a source pressure that could be independently specified. The valve was 'designed' as a check valve such that if the combustor pressure rose above the source pressure, the valve was closed. It is noted in passing that a similar bleed valve on the combustor was also investigated (e.g. flow from the combustor to atmosphere); however, the results did not indicate beneficial performance.

Before examining the combustor injection valve concept it is instructive to briefly examine a shutdown type process from the steady state idle condition. The reason for this is that the simulation results demonstrate a form of hysteresis which suggests that combustor mass flow injection will be effective. Furthermore, the results give an idea of the source pressure required for the injector. Figure 10 shows time traces for such a shutdown procedure. The wave rotor was held at the idle state for $0.25 \mathrm{sec}$. at which time the heat addition rate was abruptly set to zero. 

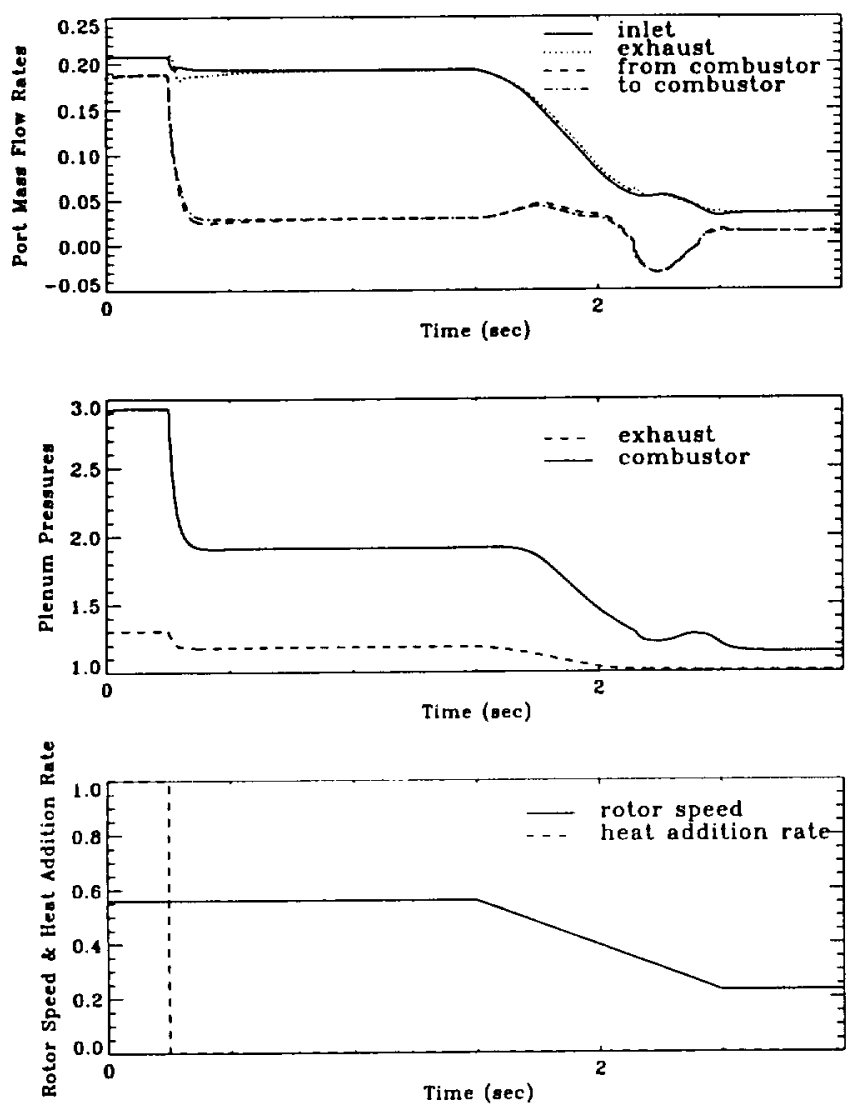

Figure 10 Time traces of flow quantities for a shutdown from idle

After reaching steady state operation, the rotor speed was slowly reduced from the idle value to the initial $23 \%$ of design value used at the start of Scenarios B, C, and D The interesting feature of this figure is that at the idle speed, and even somewhat below, the combustor loop flow direction remains positive even without heat addition. This behavior should be compared to Scenario B (Fig. 5) where the combustor loop flow is in the reverse direction prior to any heat addition. Examining Fig. 10 it can be seen that during the interval where the combustor loop flow is in the forward direction, after the heat addition has been removed, the combustor pressure is higher at any rotor speed than the combustor pressure at the same speed in Fig. 5. Furthermore, the pressure is higher than the wave rotor inlet pressure. This observation suggests that the combustor loop flow might be induced to move in the proper direction during startup if the following procedure were employed. The rotor is first accelerated to the idle speed as in Scenario B. The inlet pressure is assumed to be at the same value of 1.3 times atmosphere used in the other simulations. The combustor injector valve described above is then opened with an external source pressure corresponding to the combustor pressure in Fig. 10. Once the combustor loop flow is moving in the correct direction, the valve will shut and the combustor can be ignited. This process is illustrated in Fig. 11. The simulation commenced after the rotor had been accelerated to the idle speed of $56 \%$ of design and reached steady state. At 0.25 seconds the injector valve was instantaneously opened to a position such that the cross sectional area
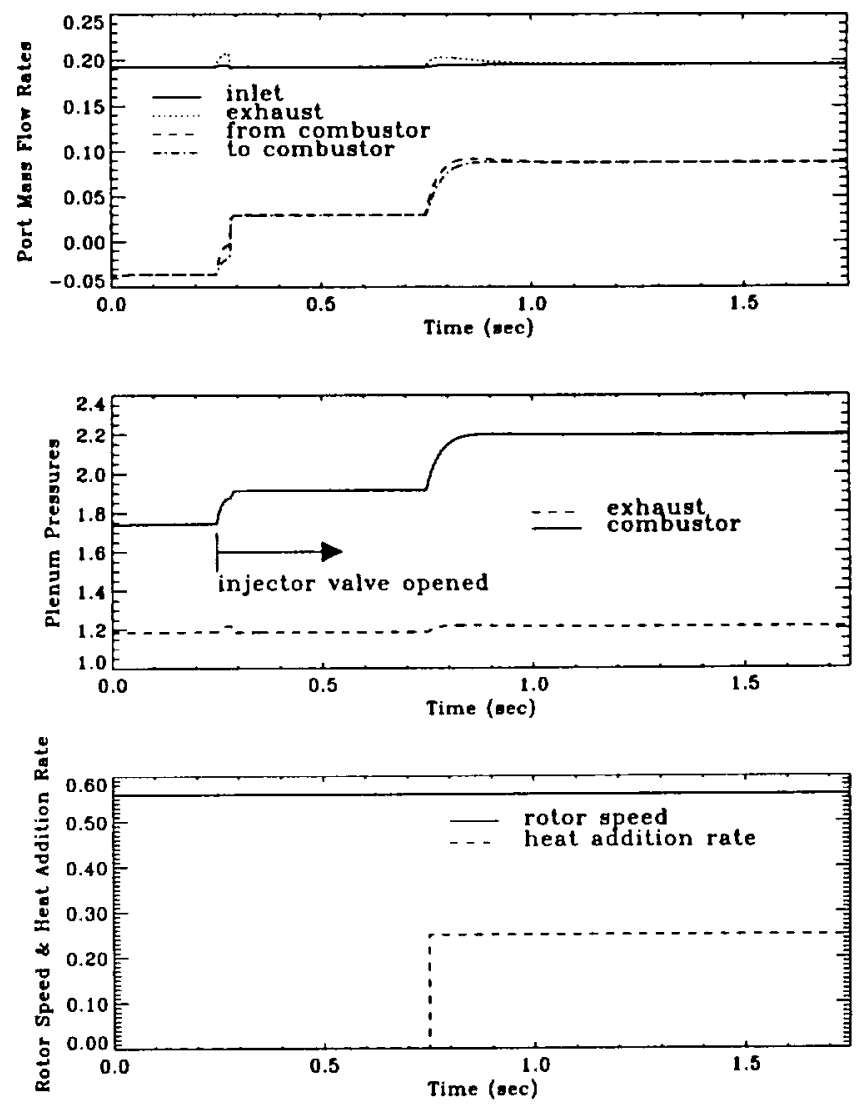

Figure 11 Time traces of flow quantities for Scenario E: Combustor Plenum Mass Injection

was $16 \%$ of the exhaust valve area $\left(3.6 \mathrm{~cm}^{2}\right)$. The source pressure for this injector was, from Fig. 10, approximately 1.91 times atmospheric. Since the injector valve was assumed to be a check valve, it was closed when the combustor pressure exceeded this value. The source temperature was chosen to be $289 \mathrm{~K}(520 \mathrm{R})$. At $0.75 \mathrm{sec}$. the heat addition rate was stepped up from zero to the lightoff value of $1.2 \%$ of design ( $25 \%$ of idle). It is seen that the combustor injector did, indeed, induce forward combustor loop flow and that the subsequent lightoff process was smooth with forward flow maintained through the combustor. The calculated total mass flow through the combustor injector valve during this transient was $0.0024 \mathrm{~kg}(0.0052 \mathrm{lbm})$. This is a low value and could conceivably be supplied in an engine environment by a small tank of stored compressed air.

\section{Scenario F: Variable Combustor Port Timing}

Since the reversed combustor loop flow problem results from wave patterns established by the port locations, it is natural to consider solutions which modify their position. Although a systematic study of the flowfield sensitivity to the location of each port was not performed, it was found that changing the location of the opening for the port leading from the combustor could affect a change in the combustor loop flow direction. This is illustrated in Fig. 12. This simulation commenced with the same boundary conditions as Scenario E (i.e.56\% of design 

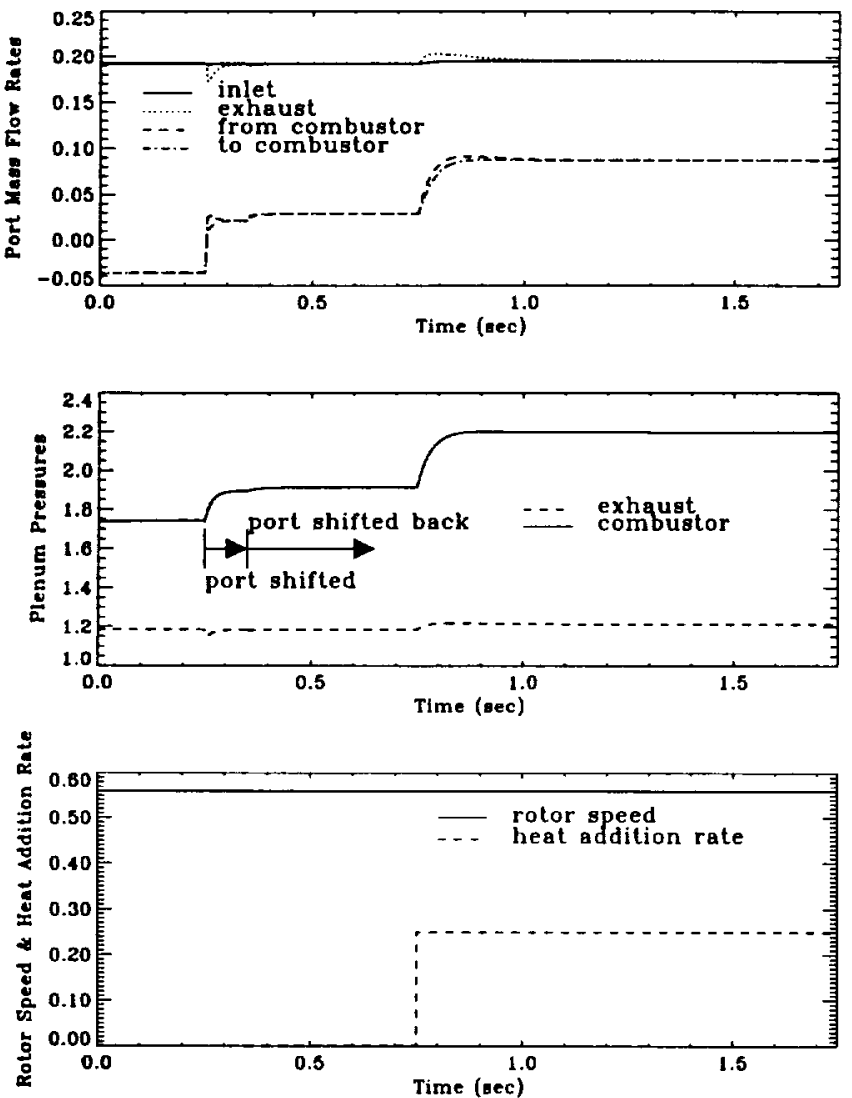

Figure 12 Time traces of flow quantities for Scenario F: Variable Combustor Port Timing

speed, inlet pressure 1.3 times atmosphere). At 0.25 seconds the position at which the port coming from the combustor opens was instantaneously shifted in the direction of rotation by 21.1 degrees. At 0.35 seconds it was instantaneously shifted back to the original location. From there the combustor lightoff procedure was identical to Scenario D, with similarly smooth results. This lightoff procedure appears promising as long as the complexity associated with blocking off a portion of the port coming from the combustor is not prohibitive.

\section{CONCLUDING REMARKS}

The starting process for a four-port through-flow wave rotor was demonstrated using a multipassage, one dimensional numerical simulation. It was found that, without augmentation to the configuration shown in Figs. 2 and 3, the wave rotor would exhibit reversed flow in the combustor loop. Once started, the flow reversal could be eliminated by increasing the heat addition rate to the system; however, this is undesirable since it necessitates an auxiliary flame source (conventional combustors cannot operate with reversed flow). It was found that the reversed flow scenario could be avoided entirely by accelerating the rotor as the combustor is ignited (i.e. at lightoff). Whether or not this is a practical starting scenario is unknown. If the combustor could deliver a highly controllable flame down to very small values of heat addition to the system, then such a technique could work. Given the current combustor technology, it is more likely that the lightoff process requires a minimum amount of heat addition, which is relatively substantial. In this case, it appears that such a technique requires a rather large motor in order to rapidly accelerate the wave rotor. Furthermore, it is unlikely that a required transient process is an acceptable procedure.

A more promising startup scenario was obtained when an injector valve was added to the simulation combustor plenum. In this case, forward flow could be induced in the combustor loop prior to lightoff and the lightoff could proceed without incident. The required driving pressure for this valve was higher than the 1.3 times atmospheric inlet pressure. In an engine environment, this would necessitate an external supply of air; however, the total valve mass flow necessary to establish forward combustor loop flow was quite small suggesting that the external air supply could consist of a small pressure vessel which would be recharged from compressor bleed during normal engine operation.

A second successful start scenario was obtained when the location of the opening for the port leading from the combustor was allowed to shift. Shifting it in the rotational direction induced forward flow through the combustor loop. Whether or not the mechanical difficulty associated with a moveable port is practical is unknown.

It is recognized that there are many other modifications which could be considered in order to make this particular type of wave rotor start reliably. Furthermore, it is noted that other types of topping cycles (e.g. four-port, reverse-flow, five-port, etc.) may not exhibit combustor loop flow reversal, and hence may be inherently easier to start. The intentions of this paper were to demonstrate the potential problems that exist for a four-port, through-flow wave rotor, to describe some reasonable, preliminary solutions, and to illustrate the insight that may be obtained using a numerical simulation such as the one described.

\section{REFERENCES}

Klapproth, J. F., 1960, "The Wave Engine Project," General Electric Quarterly Reports., R60FPD284, R60FPD419, \& R60FPD585.

Moritz, R., 1985, "Rolls-Royce Study of Wave Rotors 1965-1970," eds. Shreeve, R. P. and Mathur, A., Proceedings of the 1985 ONR/NAVAIR Wave Rotor Research and Technology Workshop, Report \# NPS-67-85-008, Naval Post Graduate School, Monterey, CA.

Paxson, D. E., 1995a, "A Numerical Model for Dynamic Wave Rotor Analysis," AIAA \# 95-2800.

Paxson, D. E., 1995b, "Recent Improvements to and Validation of the One Dimensional NASA Wave Rotor Model," NASA TM 106913.

Paxson, D. E., 1995c, "Comparison Between Numerically Modeled and Experimentally Measured Wave-Rotor Loss Mechanisms," AIAA Journal of Propulsion and Power, Vol. 11, pp. 908-914.

Thayer, W., J. 1985, "Energy Exchanger Performance Measurements and Projections," eds. Shreeve, R. P. and Mathur, A., Proceedings of the 1985 ONRNAVAIR Wave Rotor Research and Technology Workshop, Report \# NPS-67-85-008, Naval Post Graduate School, Monterey, CA.

Welch, G. E., Jones, S. M., and Paxson, D. E., 1995, "Wave RotorEnhanced Gas Turbine Engines," AIAA-95-2799.

Wilson, J., and Paxson, D. E., 1995, "Optimization of Wave Rotors for Use as Gas Turbine Engine Topping Cycles," SAE \# 951411.

Zehnder, G., Mayer, A., and Matthews, L., 1989, "The Free Running Comprex," SAE \# 890452. 



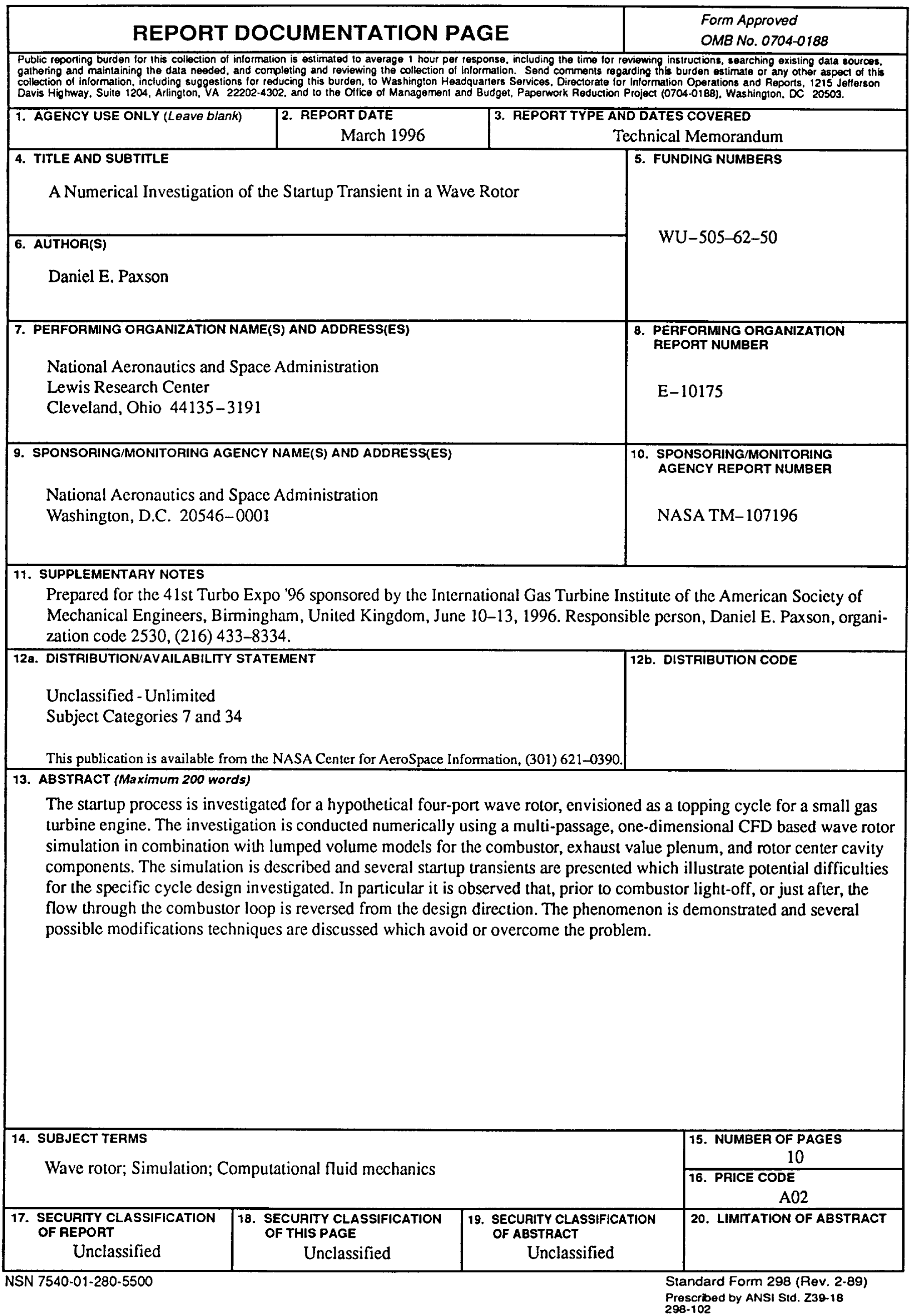




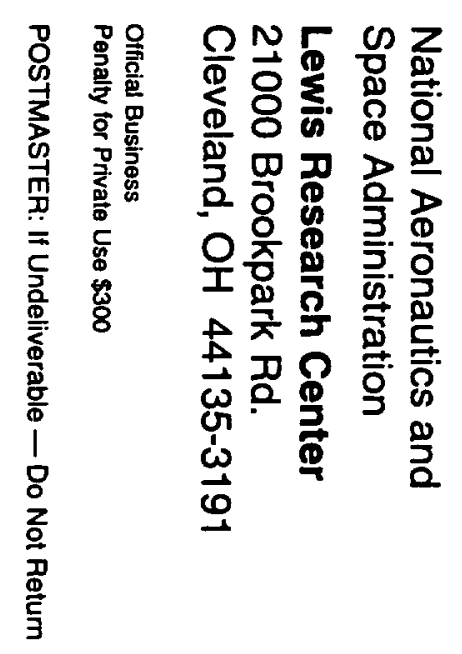

